\title{
Erhöhtes Suizidrisiko kurz nach Klinikentlassung
}

Fragestellung: Untersucht wurde das kurzfristige Suizidrisiko nach stationärer Entlassung bei Patienten mit unterschiedlichen psychiatrischen Hauptdiagnosen im Vergleich zu Patienten ohne psychiatrische Entlassdiagnose und zur Allgemeinbevölkerung.

Hintergrund: Psychiatrische Patienten weisen kurz nach der stationären Entlassung ein deutlich erhöhtes Suizidrisiko auf. Die meisten der bisher durchgeführten epidemiologischen Studien konzentrierten sich jedoch auf nur eine psychiatrische Erkrankung, sodass bisher wenig darüber bekannt ist, inwieweit sich das kurzfristige Suizidrisiko von Patienten mit unterschiedlichen psychiatrischen Diagnosen unterscheidet.

Patienten und Methodik: Im Rahmen einer retrospektiven Kohortenstudie wurden die Daten von 1.861.194 stationären $\mathrm{Pa}$ tienten im Alter zwischen 18 und 64 Jahren des staatlichen Gesundheitsfürsorgeprogramms „Medicaid“ in den USA erfasst. Davon wiesen 770.643 eine psychiatrische Entlassdiagnose auf. Primärer Studienendpunkt war der vollendete Suizid innerhalb von 90 Tagen nach Entlassung. Die Patienten wurden auf Basis der Hauptentlassdiagnose in depressive Störung, bipolare Störung, Schizophrenie, Substanzabhängigkeit, andere psychische Erkrankungen und keine

Olfson $\mathrm{M}$, Wall $\mathrm{M}$, Wang $\mathrm{S}$ et al. Short-term suicide risk after psychiatric hospital discharge. JAMA Psychiatry 2016; 73 : $1119-26$ psychiatrische Entlassdiagnose unterteilt. Bestimmt wurden die Suizidraten der entlassenen Patienten, aufgeteilt nach Hauptentlassdiagnose und Geschlecht.
Ergebnisse: Die Suizidrate der Patienten mit psychiatrischer Entlassdiagnose war etwa 15-mal höher als bei Patienten, die ohne psychiatrische Diagnose aus der stationären Behandlung kamen $(178,3$ vs. 11,6). Die höchste kurzfristige Suizidrate zeigte sich bei Patienten mit depressiver Störung $(235,1)$, gefolgt von Patienten mit bipolarer Störung $(216,0)$. An dritter Stelle standen Patienten mit einer Schizophrenie $(168,3)$. In der Gruppe mit psychiatrischer Entlassdiagnose war die Suizidrate der Männer fast zweimal höher als die der Frauen (238,3 vs. 130,2). Zudem zeigte sich in dieser Gruppe insbesondere bei Männern ein deutlich erhöhtes kumulatives Suizidrisiko wenige Tage nach der Entlassung. In der Gruppe mit psychiatrischer Entlassdiagnose wiesen die Patienten im Alter zwischen 45 bis 64 Jahren im Vergleich zur Referenzgruppe der 18- bis 34-Jährigen ein 1,8-fach höheres Risiko eines Suizids 90 Tage nach stationärer Entlassung auf (Hazard Ratio, HR] 1,8). Männer dieser Gruppe hatten ein 2,3-fach höheres Risiko als Frauen (HR 2,3), Patienten mit einer depressiven Störung ein zweifach höheres Risiko als Patienten mit der Diagnose einer Substanzabhängigkeit (HR 2,0). Zudem zeigte sich ein 1,7-fach erhöhtes kurzfristiges Suizidrisiko bei Patienten, die sich in 180 Tagen vor stationärer Aufnahme nicht in ambulanter Behandlung befanden (HR 1,7).

Schlussfolgerungen: Psychiatrische Patienten, insbesondere Patienten mit der Diagnose einer affektiven Störung, haben ein deutlich erhöhtes Suizidrisiko kurz nach stationärer Entlassung. Ein erhöhtes kurzfristiges Suizidrisiko ist zudem mit höherem Alter, männlichem Geschlecht und nicht vorhandener ambulanter Behandlung 180 Tage vor stationärer Aufnahme assoziiert.

\section{Systematische Evaluation von Suizidalität vor stationärer Entlassung}

In dieser epidemiologischen Studie mit großer Studienpopulation konnte eindrücklich gezeigt werden, dass psychiatrische Patienten, insbesondere kurz nach stationärer Entlassung, ein deutlich erhöhtes Suizidrisiko im Vergleich zu Patienten ohne psychiatrische Entlassdiagnose aufweisen. Dies gilt vor allem für Patienten mit affektiven Störungen. Wie bereits vielfach in der Literatur beschrieben, zeigte sich auch hier ein deutlich erhöhtes Suizidrisiko bei Männern. Zudem scheinen diejenigen Patienten, die vor dem stationären Aufenthalt nicht in eine ambulante Behandlung eingebunden waren, ebenfalls ein erhöhtes Suizidrisiko nach der Entlassung zu haben.

Einschränkend muss erwähnt werden, dass die Teilnehmer der Studie nur Patienten aus dem „Medicaid Program“ waren, einem staatlich organisierten Gesundheitsfürsorgeprogramm für Personen mit geringem Einkommen oder Behinderungen. Nicht betrachtet wurden daher zum Beispiel privatversicherte Patienten. Darüber hinaus fehlte die Betrachtung älterer Patienten. Andere bekannte Risikofaktoren für Suizidalität, wie Suizidversuche in der Vorgeschichte oder Suizide in der Fami- lie, wurden ebenfalls nicht untersucht. Die Ergebnisse unterstreichen die Bedeutung einer systematischen Evaluation von Suizidalität vor der Entlassung aus stationärer Behandlung, einschließlich der relevanten Risikofaktoren. Darüber hinaus weist die Studie auf die Bedeutung der ambulanten Weiterbehandlung hin, die bereits im Rahmen des Entlassmanagements zusammen mit dem Patienten organisiert werden sollte.

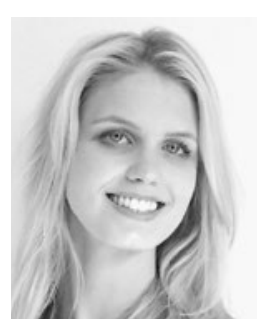

Dr. med. Marie-Jocelyne Walter, Mainz

Klinik für Psychiatrie und Psychotherapie,

Universitätsmedizin Mainz

E-Mail: marie-jocelyne.walter@

unimedizin-mainz.de 